\title{
A Fissile Ripple Spreading Algorithm to Solve Time-Dependent Vehicle Routing Problem via Coevolutionary Path Optimization
}

\author{
Wen Xu iD and JiaJun Li \\ School of Management, Northwestern Polytechnical University, Xi'an 710072, China \\ Correspondence should be addressed to Wen Xu; 18078115229@126.com
}

Received 2 April 2020; Revised 30 June 2020; Accepted 8 July 2020; Published 1 August 2020

Academic Editor: Weiwei Qi

Copyright (c) 2020 Wen Xu and JiaJun Li. This is an open access article distributed under the Creative Commons Attribution License, which permits unrestricted use, distribution, and reproduction in any medium, provided the original work is properly cited.

\begin{abstract}
The time-dependent vehicle routing problems have lately received great attention for logistics companies due to their crucial roles in reducing the time and economic costs, as well as fuel consumption and carbon emissions. However, the dynamic routing environment and traffic congestions have made it challenging to make the actual travelling trajectory optimal during the delivery process. To overcome this challenge, this study proposed an unconventional path optimization approach, fissile ripple spreading algorithm (FRSA), which is based on the advanced structure of coevolutionary path optimization (CEPO). The objective of the proposed model is to minimize the travelling time and path length of the vehicle, which are the popular indicators in path optimization. Some significant factors usually ignored in other research are considered in this study, such as congestion evolution, routing environment dynamics, signal control, and the complicated correlation between delivery sequence and the shortest path. The effectiveness of the proposed approach was demonstrated well in two sets of simulated experiments. The results prove that the proposed FRSA can scientifically find out the optimal delivery trajectory in a single run via global research, effectively avoid traffic congestion, and decrease the total delivery costs. This finding paves a new way to explore a promising methodology for addressing the delivery sequence and the shortest path problems at the same time. This study can provide theoretical support for the practical application in logistics delivery.
\end{abstract}

\section{Introduction}

The vehicle routing problem (VRP) and its extended variants have recently received great attention in many fields, which are required to improve delivery efficiency and reduce the delivery costs of logistics companies [1-6]. In its basic form, some essential elements are often used to construct the foundation of the VRP resolution, for instance, several depots and a set of customers [7]. Each customer is located in a different geographic position and demands a specific amount of goods, which are carried to the designative destinations by a fleet of vehicles [8]. The vehicles will depart from the depots and finally return to the same depots, if the given delivery assignments are completed. The typical constraints of VRP are to pose a strict requirement on the vehicle travelling trajectory. That is, all matched sites can be visited only once along with the planned vehicle route. The common optimization objective of VRP is to seek the optimal delivery sequence and the shortest path performed by the specific vehicle. It requires that all customers can receive the associated goods, aiming at cutting down the related travelling time and path length of the overall routes.

Due to the boosting number of vehicles in urban networks, regular traffic congestion has been a common phenomenon that imposes some negative impacts on social development and daily life [9]. Especially for the logistics industry, several inevitable challenges are brought to impede the further research of the VRP and its multiple variants. How to effectively solve VRP plays a considerable role in minimizing delivery costs and ecological environment protection [10]. For example, the daily traffic congestions may cause huge uncertainties for vehicle route planning, when the vehicles intend to travel across a planned path with shorter length but will waste a long time waiting at the 
blocked areas [9]. The fuel consumption highly depends on the length and travelling time of the route. Hence, traffic congestion tends to result in high fuel consumption and air pollution with $\mathrm{CO}_{2}$ emissions because the engine must keep running during waiting periods. According to reliable data reported by the International Energy Agency, the transportation sector has contributed to the second-largest $\mathrm{CO}_{2}$ emissions in 2015 [11]. In recent decades, how to reduce carbon emissions and fuel consumption has become a significant issue that should be paid more attention to VRP [12-16]. Under the pressure of additional costs and environmental protection, the current concerns of logistics companies are to exploit an appropriate approach for addressing the VRP [17-19].

Most of the research studies investigated the VRP proposed some ideal hypotheses. For example, the vehicle travel speed between two nodes is assumed to be fixed, which means that the travelling time is simply regarded as a constant value. Under the background of the time-dependent vehicle routing problem (TDVRP), it is impractical to be supposed in the real world because the vehicle speeds may vary with the changes of traffic condition. In other words, the travel speed between two nodes is timedependent and usually relies on the starting time. When the vehicle reaches a node, the related link and node that will be visited soon may transform into blocked from the accessible state, influenced by sudden traffic congestion. As a result, an extra detour strategy or waiting behaviour both make the travelling time very long, even though the planned path possesses the shortest length. The longer path between two sites may not experience traffic congestion during the same period. A long path is not consistent with large travelling time [20]. Therefore, the vehicle route should take into account the changes in traffic conditions. Malandraki was the first to investigate TDVRP via exploring the impacts of traffic congestion on vehicle speeds [21]. Eglese et al. designed a timetable of the shortest paths based on a practical network, to present the expected route distance and travel time between two nodes [22]. Donati et al. developed an advanced multi-ant-colony system to minimize the total travel time, during a fleet of vehicles travelling at time-dependent speeds with a fixed capacity [23]. Kok et al. employed a modified Dijkstra algorithm and dynamic programming heuristic to implement an appropriate strategy to avoid traffic congestion in TDVRP [24]. Huang et al. developed the TDVRP with path flexibility (TDVRPPF) under the stochastic traffic conditions and gave the path decisions that relied on the departure time [25]. Kuo adopted simulated annealing to minimize the fuel consumption used in the route for TDVRP [26]. Ma et al. proposed a combined order selection to address the TDVRP with time windows for perishable product delivery [27]. Taş et al. studied the TDVRP considering soft time window and stochastic travel time, and applied tabu search and adaptive large neighbourhood search to prove the effectiveness of the proposed model [28]. Andres Figliozzi proposed a promising algorithm that can well solve TDVRP with a soft or hard time window without any alteration [29].
To address TDVRP in the real world, this study aims to minimize the travelling time and path length of the route by solving the optimal delivery sequence and the best path at the same time. A novel fissile ripple spreading algorithm (FRSA) was proposed to find the optimal solution by avoiding daily traffic congestion in advance, or waiting to pass through a rapidly shrinking congestion. The coevolutionary path optimization (CEPO) technology was introduced to apply in the proposed method, according to the significant characteristic of traffic operational rules and delivery demand. The effectiveness of the proposed approach was illustrated in two sets of simulation experiments. The results proved that the proposed FRSA can effectively reduce the travelling time and path length of the vehicle route, which can decline fuel consumption and avoid regular traffic congestion.

The rest of this study is organized as follows: Section 2 explains the problem description and the details of CEPO technology introduced in this work. Section 3 presents the basic idea of the proposed FRSA and shows how to integrate CEPO into it. Section 4 shows the experimental results by comparing the performance of FRSA with another compound method. Section 5 discusses some possible reasons for the outstanding results derived from FRSA and indicates its potential application. Section 6 outlines the conclusions of this work.

\section{Coevolutionary Path Optimization}

2.1. Problem Description. As a critical solution to reduce travel time and fuel consumption, TDVRP has been studied in extensive fields, which provides a great potential alternative to boost delivery efficiency and decrease economic cost. However, most of the existing studies ignored the practical network topology and separate the essential relationship between the shortest path of two sites and delivery sequence. Generally speaking, the delivery sequence decision is greatly affected by the weight values between two sites, which are obtained from the shortest path optimization. On the other hand, if a vehicle set out from a site at different periods, it tends to encounter different traffic conditions, and the shortest paths are likely to be changed. One can see that the path optimization results are also influenced by the delivery sequence. Furthermore, few studies focus on how to design a general algorithm to consider traffic congestion. Traffic congestion is the most common phenomenon in urban roads, which tends to cause a lot of time delay for vehicles as well as fuel consumption, along with some potential damage for emergency rescue. Therefore, although some encouraging progress has been made, developing a better intelligent algorithm with excellent optimization performance and good applicability is less successful and still requires further research. To overcome these research gaps, this paper firstly proposed a universal approach called FRSA to address TDVRP, considering dynamic traffic congestion and the coupling relationship between the shortest path and delivery sequence.

Thanks to the foundation offered by the previous research, the proposed TDVRP in this study aims to make the 
actual delivery trajectory optimal, and it can be separated into two important issues. The one is to propose an appropriate path optimization method to find out the shortest path between two sites. The other is to exploit a feasible algorithm to determine the best delivery sequence with small travelling time and short path length on account of the path optimization method. In other words, the goal is to minimize the total travel time and path length by optimizing the vehicle delivery order and the delivery paths between any two sites.

According to the formal description of TDVRP in previous studies, several crucial rules should be established. A series of predetermined sites must be reached by a specific vehicle that is located in a single depot. The locations and the demands of each site are known, while the location and capacity of the related depot are given as well. Due to the complicated effects of traffic flow and road geometry, vehicle speeds may vary over time in a road section [30-33]. Additionally, when the vehicle travels on different roads or different driving directions, it tends to encounter various speeds and traffic conditions. To clarify the application scope of this study, two assumptions must be made. (1) The vehicle is allowed to wait at signalized intersections or congested links, and the delay caused by this inevitable procedure will be contained in total travel time. (2) The vehicle can get access to future traffic data from the traffic information prediction platform or big data prediction algorithm. The future traffic data include congestion scope, the average delay of signalized intersections, and average traffic speeds of all roads. (3) Any change in the routing environment will eventually be reflected by the certain variation of traffic speeds in related roads.

2.2. Basic Idea of CEPO. In this paper, an advanced framework of CEPO is introduced to improve the path optimization algorithm, which concentrates on how to find out the best path between different sites. As a newly emerging approach, CEPO is firstly proposed by $\mathrm{Hu}$ et al. who have certified its outstanding performance and feasibility in discrete urban networks [34]. Moreover, another advanced variant timing coevolutionary path optimization (TCEPO) was developed to prove the effectiveness of CEPO by several comparative simulation experiments [35]. Due to its coevolutionary optimization mechanism, it can achieve a global optimal result as long as the given prediction data are precise enough. The prediction data in this study are obtained from those intelligent prediction algorithms, which are used to predict the short-term traffic flow or traffic speed based on historical traffic data. The prediction algorithms tend to adopt machine learning theory to predict traffic dynamics and usually achieve more than $90 \%$ of mean prediction accuracy [36-38]. Some of them can achieve higher prediction accuracy, even more than 95\% [38]. These encouraging results can provide the predictive traffic data, which are assumption basis for the proposed method. To explain how it can implement a superior optimization process, the basic idea of CEPO is illustrated in Figure 1.

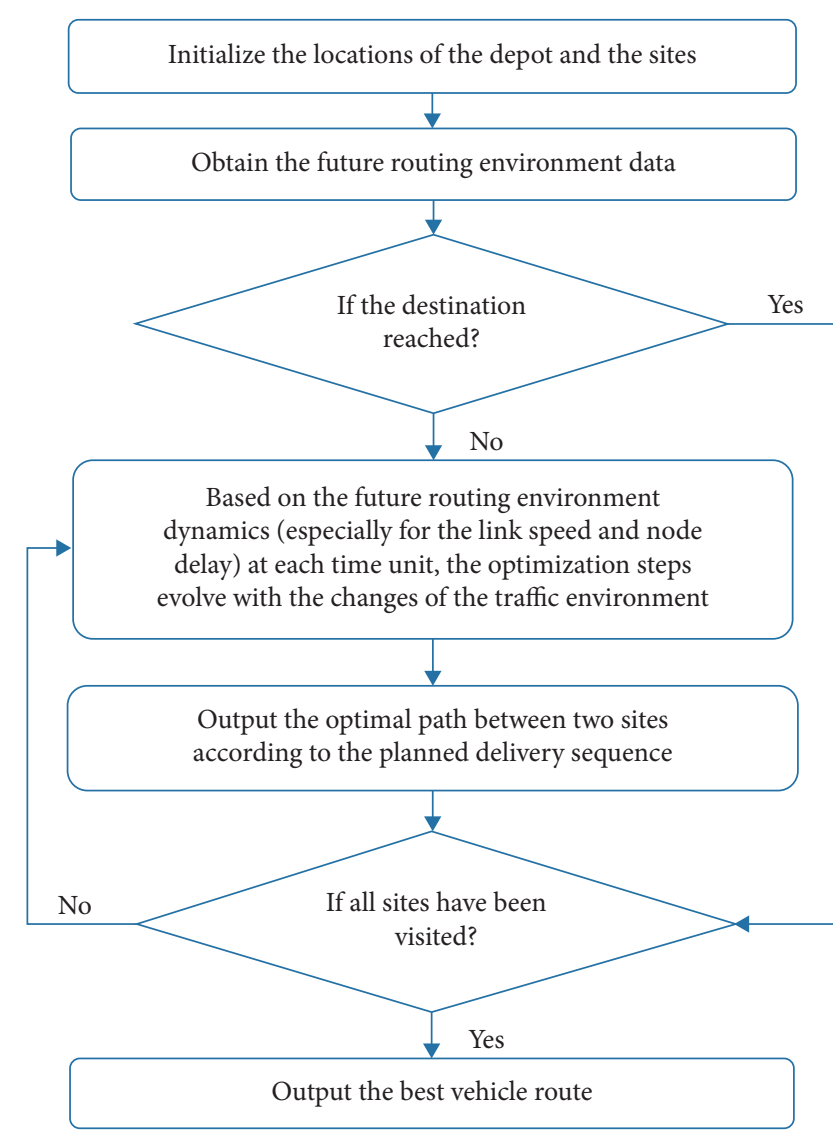

FIGURE 1: The framework of the optimization process for the CEPO technology.

The definition of CEPO is, under the predicted traffic environment dynamics, each time-unit-oriented optimization step coevolves with the traffic speed variation of associated links once the traffic environment changes in routing networks, to make actual travelling trajectory optimal in a single run. All optimization steps will be carried out before the vehicle sets out from the depot. Unlike other mainstream research studies based on online reoptimization (OLRO), CEPO has more advantages in utilizing future traffic information and optimization accuracy. This is because the future routing environment dynamics are considered into the optimization procedure, which brings an extra superiority to know the evolutionary trend of routing environment in advance. As a result, it can help a vehicle to avoid wrong detour decisions or escape from congestion areas in advance. No matter which kind of roles it plays, CEPO poses a positive effect on the best route decisions that can help vehicle travel across the temporary congestion areas as fast as possible.

2.3. Mathematic Expression of CEPO. It is assumed that the predictable routing environment dynamics and vehicle delivery sequence are both given, CEPO aims to address the minimization problem in terms of time cost. Travelling time is usually a major concern in path optimization, particularly in the dynamic urban routing environment [39, 40]. Moreover, 
as explained in Section 2.3, CEPO technology is a time-unitoriented optimization methodology. Thus, it is an effective way to use travelling time as the optimization objective of CEPO. The objective function can be established as follows:

$$
\min F_{C}=\sum_{k=1}^{n+1} f_{k}\left(P_{k}, L\left(P_{k}\right)\right),
$$

which is subject to the following equations:

$$
\begin{aligned}
& f_{k}\left(P_{k}, P_{k}(i+1)\right)=D_{t}\left(P_{k}(i)\right) \\
& \quad+C_{t}\left(P_{k}(i), P_{k}(i+1)\right), \quad i=1, \ldots, L\left(P_{k}\right)-1, \\
& f_{k}\left(P_{k}, 1\right)=0,
\end{aligned}
$$

where $F_{c}$ is the function calculating the time cost of all optimization paths. $n$ denotes the number of delivery sites. $P_{k}$ denotes the $k$ th optimization path between two sites in the presupposed delivery sequence. $L\left(P_{k}\right)$ denotes the number of all nodes in path $P_{k} \cdot f_{k}\left(P_{k}, L\left(P_{k}\right)\right)$ denotes the time to travel through all nodes in path $P_{k} \cdot f_{k}\left(P_{k}, P_{k}(i+1)\right)$ denotes the time to pass through the node $P_{k}(i+1)$ when the vehicle just reaches the node $P_{k}(i) . D_{t}\left(P_{k}(i)\right)$ denotes the time delay to cross over the node $P_{k}(i)$ in path $P_{k}$ at time $t$. $C_{t}\left(P_{k}(i)\right.$, $\left.P_{k}(i+1)\right)$ denotes the travelling time associated with the link between nodes $P_{k}(i)$ and $P_{k}(i+1)$ at time $t$.

In this section, the objective function in (1) is defined as minimizing the total travelling time of the complete delivery loop. Equations (2) and (3) clearly show the calculation derivation of the travelling time, consisting of the time costs that pass through the nodes and links, respectively. It is believed that the time cost of a subpath in $P_{k}$ tends to be dependent on when the subpath is to be travelled through, which is often overlooked in other studies. Thus, if the routing environment dynamics are predictable, one-off optimization will be prospectively conducted to seek the best path $P^{*}$ in the time-varying traffic environment before the vehicle starts to perform the delivery assignments. This means that the reoptimization of the path $P^{*}$ required in OLRO-based methods is unnecessary to use at each time unit.

\section{Fissile Ripple Spreading Algorithm Based on Global Search}

3.1. Algorithm Origin and Development. To realize the CEPO method as mentioned in Section 2, it is essential to exploit an appropriate algorithm to adapt to the synchronous state changes of nodes and links. It has achieved a great success for deterministic and heuristic methods in solving static path optimization and time-dependent path optimization. For example, the Dijkstra algorithm, A* algorithm, genetic algorithm, particle swarm optimization algorithm, simulated annealing algorithm, and ant colony algorithm. It is unlikely to apply them to an effective realization of CEPO. One important reason is that their basic computational step is the link-oriented analysis that associates with a long period, which may cause serious scalability problems [30]. What makes things worse is that it is impossible to pre-know which link will be accessed or when the given links will be accessed. Additionally, it must be considered for the improvement of delivery strategy and the best-first search of the shortest paths between sites. According to the latest knowledge, it seems to be quite hard to synchronously perform this procedure for most methods. This is why in a time-dependent routing environment, an advanced optimization algorithm needs to be designed to achieve the CEPO, to cover all necessary changes in the route network.

FRSA is an evolutionary branch of the ripple spreading algorithm (RSA), which is first reported by $\mathrm{Hu}$ and Liao in 2016 [41]. Like other evolutionary computation algorithms, the basic idea of FRSA takes inspiration from nature and intends to imitate the ripple spreading phenomenon. The core technology of FRSA presents excellent optimality and surprisingly robustness in resolving complex problems, which is conducted by a multilevel and fissionable ripple relay race. FRSA is an agent-based, deterministic, and discrete simulation model, which provides a rare opportunity to evolve with the changes in the predicted environment at each time unit. During a single run of FRSA, it delivers a great number of ripples and the spreading speeds of ripples reflected in the changes of links to program optimization steps. Furthermore, when a ripple activates a site from the last site, the fissile mechanism is permitted to carry out more independent ripple relay races that explore optimal resolution in global research. Thus, once a single run of FRSA has been done, optimal delivery sequence and the shortest paths of given OD pairs (site to site or depot to site) can be obtained.

\subsection{Fissile Ripple Relay Race Based on Global Research.} To realize the CEPO methodology, the optimization principle of the FRSA is extended to be divided into two parts, that is, independent ripple relay race and fissile program. An original ripple relay race is applied in a static route network [42]. But in the actual application, especially for the urban network, traffic environment dynamics should be integrated into an independent ripple relay. The illustration of an independent ripple relay race is given in Figure 2.

In the ripple relay race, the initial ripple is activated to split into several ripples from the origin node, and new ripples travel toward the adjacent nodes along with the connected links. When a ripple reaches an unvisited node, it will trigger new ripples at that node, and the unvisited state will be transformed into an active state. Those new ripples will continue to spread out until arriving in the adjacent nodes, while their spreading speeds are consistent with the traffic speeds of the link. As the ripple relay race goes on, numerous ripples will be generated to compete with each other until a ripple reaches the given destination node. Eventually, the shortest path from the origin to the destination will be determined by tracking back the visiting node order, when the ripple reaches the destination firstly. Therefore, the travelling time of the shortest path between sites can be calculated as the end of an independent ripple relay race. 


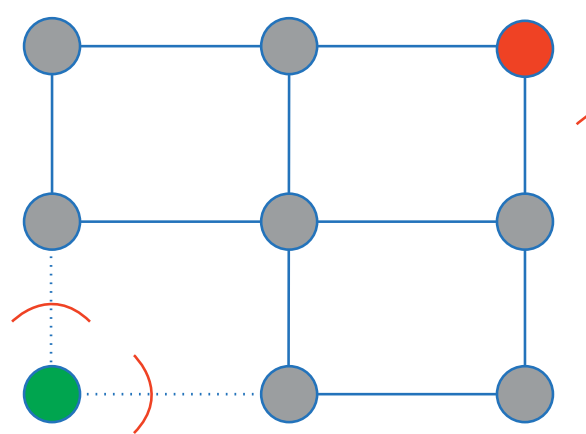

(a)

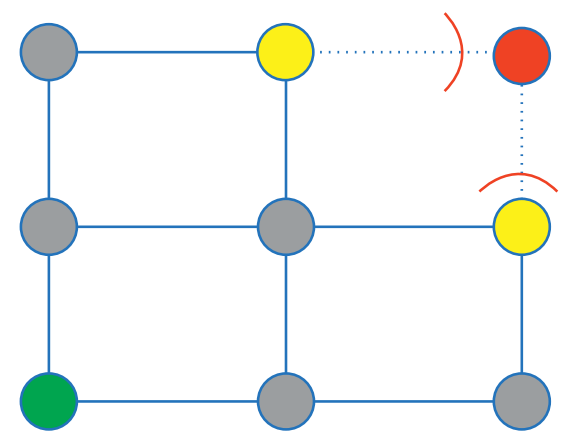

(c)

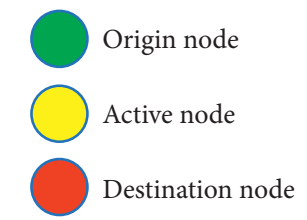

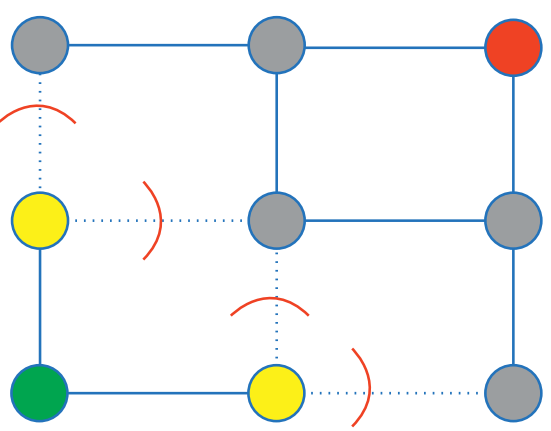

(b)

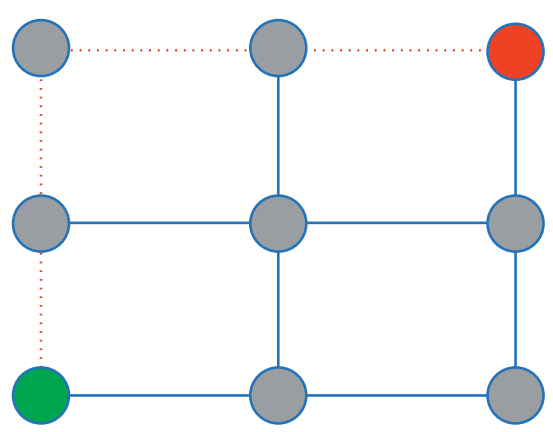

(d)

Figure 2: The illustration of an independent ripple relay race.

Although the independent relay race just offers a supportive foundation for addressing TDVRP in a dynamic routing environment, it is still less successful. It seems to be an ongoing challenge that how to decide an optimal delivery sequence with the smallest travel time cost. Hence, how to apply ripple relay race to resolve the best visiting order for all preset sites may be a question, which is worth thoroughly pondering. However, thanks to the development of computer science technologies, stronger computational ability can be used to execute big data processing. It opens a door to the fissile program that can evolve more independent relay races, to search more loop paths associated with different visiting orders.

To explore the optimal delivery sequence, it is a critical way to search for all delivery schemes in parallel by performing extensive ripple relay races, which are independent of each other. The diagram of the fissile program in FRSA is given in Figure 3. When the nearest site will be triggered by a ripple from the depot, it will generate a unique list recording activation order of the site. At the same time, a new ripple relay race starts to run at the site. All ripples from the new ripple relay race will not be affected by any ripple from the previous one. In other words, the ripples of different relay races are independent of each other, although they may arrive in the same nodes or links simultaneously. All ripple relay races run in the original size network. What is more

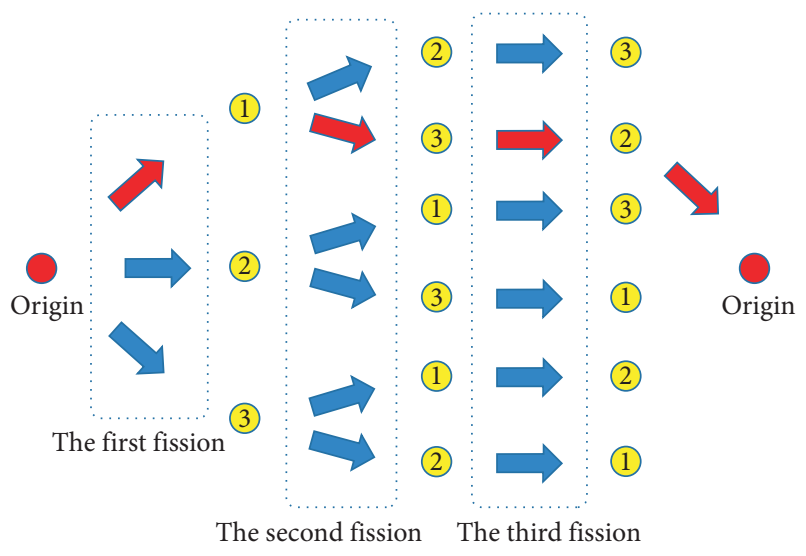

The optimal route

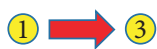

(2)

Ripple relay race

The fastest solution

FIgURE 3: The schematic diagram of the fissile program in FRSA.

important, the previous ripple relay race will continue to program, even if it has given rise to a new ripple relay race at the unvisited site. The other ripples from the previous relay race still own similar ability to trigger other sites, as long as these sites are not contained in the list of activity orders 
recorded by the associated ripples. This strict rule of FRSA indicates that a site will not be triggered twice in the same ripple relay race but remains accessible to others. As time goes on, more and more independent ripple relay races are conducted to evolve with the routing environment dynamics. The optimization of FRSA will be terminated, when all sites are recorded in the list of activation order and the related ripple finally reaches the depot in the first place. The optimal loop path can be gained by a single run of FRSA after a large number of computations for time-unit-oriented analysis.

\subsection{Realization of FRSA considering the CEPO Technology.} To make the actual loop path optimal, it is crucial to permit ripples to wait at a node that represents a signalized interaction in practical application. The waiting behaviour of ripples reflects that the vehicles have to obey legal traffic rules, such as the restriction of red light and forward vehicles. Another condition is that the ripples can wait at a link or a node when they are temporarily blocked due to the evolution of the congestion area. However, this waiting behaviour provides a potential likelihood of waiting at a link or node until they become accessible again, which may take less time than travelling around the block area. It can impose a positive impact on avoiding the wrong detour, which is helpful to find out the shortest path with the smallest travelling time. Therefore, to extend the FRSA to realize the CEPO technology, it needs to introduce a waiting behaviour of ripple into the relay race, to imitate the real performing actions of vehicles in the urban roads.

To understand the outline of FRSA, some variables need to be calibrated to explain how it works. Let $N$ denote the total number of nodes in the network; $n$ denotes the number of sites. For the sake of simplicity but without losing generality, this paper supposes that there are one hundred nodes, three sites, and one depot in the network. Let node 1 represent depot. $m$ is the number of adjacent nodes for any node and sets $m=4$, which means each node is connected to four nodes. In a ripple relay race, node $i$ can be triggered with unlimited times and its ripple is called ripple $i$, which can split into several arcs spreading to adjacent nodes. $S_{r}(i)$ is the state of the ripple $i, S_{r}(i)=1,2,3$ means ripple $i$ remains inactive, active, and waiting, respectively. $r(i, j)$ is the radius of ripple arcs from node $i$ to node $j(i \neq j) . V_{t}(i, j)$ denotes the spreading speed of ripple arc from node $i$ to node $j$ at time $t$, which is related to expand the associated arcs radius $r(i, j) . F_{r}(i)$ denotes a crucial variable that records which ripple triggered the node $i$. For instance, $F_{r}(i)=j$ means node $i$ is triggered by the ripple from node $j$, which offers detailed information to track back the travelling trajectory. When the ripple relay races are terminated, the optimal loop path $P^{*}$ will be determined by iterative deduction of $F_{r}(i)$, and the expression is illustrated in the following equation:

$$
P^{*}(i)= \begin{cases}1, & \text { if } i=L\left(P^{*}\right), \\ F_{r}\left(P^{*}(i+1)\right), & \text { if } i<L\left(P^{*}\right)\end{cases}
$$

To avoid the repeated activation of the sites in the same ripple relay race, $M_{r}(i)$ is used to record the existing travelling trajectory of the ripple $i$ via tracking back $F_{r}(i)$. One can see that any site will not be repeated on each $M_{r}(i)$ except for the depot, which is the origin as well as the destination. In the real world, the spreading speed of ripple $i$ is subject to the speed limit of the urban roads. Thus, $V_{t}(i, j)$ must satisfy the following constraints, which are given in the following equations:

$$
\begin{aligned}
& 0<V_{t}(i, j) \leq V_{\lim }(i, j), \\
& V_{t}(i, j) \times t_{\text {unit }} \leq L(i, j),
\end{aligned}
$$

where $V_{\lim }(i, j)$ is the maximal speed limit of the link $(i, j)$. $t_{\text {unit }}$ is the time unit that is small enough to capture the major dynamics of the routing environment. $L(i, j)$ is the length of the link $(i, j)$. As time goes on, the arc radius of the active ripple will be updated according to the associated spreading. The calculation formula of the arc radius is shown in the following equation:

$$
r(i, j)=r(i, j)+V_{t}(i, j) \times t_{\text {unit }} .
$$

If the node $i$ is reached by the ripple $j$ before the ending of a time unit, the new ripple $i$ would continue to spread out in the remaining time $t_{\mathrm{re}}$. The spreading radius of arcs from the ripple $i$ to its adjacent node $k$ in the remaining time $t_{\text {re }}$ can be obtained according to the following equations:

$$
\begin{gathered}
t_{\mathrm{re}}=\frac{r(j, i)-L(j, i)}{V_{t}(j, i)}, \\
r(i, k)=r(i, k)+V_{t}(i, k) \times t_{\mathrm{re}},
\end{gathered}
$$

where $t_{\mathrm{re}}$ is the remaining time in a time unit when a ripple arrives at a node. $r(i, k)$ denotes the spreading radius of arcs from the ripple $i$ to its adjacent node $k$ in the remaining time $t_{\text {re. }}$.

The flowchart of the FRSA based on the CEPO methodology is demonstrated in Figure 4. One can see that Step 1 to Step 3 well describe how to construct a simulation network and initialize some parameters as the prior preparation. Step 4 defines the termination condition of the ripple relay race; that is, all sites are visited by a specific ripple that finally returns to the depot. Step 5 indicates the function that converts the state of the node from waiting into active if the node is accessible. Step 6 gives the derivation formula of the arc radius for an active ripple. Step 7 means that the remaining time will be used to develop the arc radius of the new active ripple, which is triggered by other ripples before the ending of a time unit. Step 8 demonstrates the requirement of a waiting behaviour at a triggered node, while it is blocked. Step 9 updates the state of the nodes to inactive again, when their all ripples have reached adjacent nodes. Step 10 judges whether FRSA needs to start a new ripple relay race from a node. Step 11 explains the mandatory operation rules for a new ripple relay race. Step 12 outputs the optimal travelling trajectory based on complete $M_{r}(1)$. 


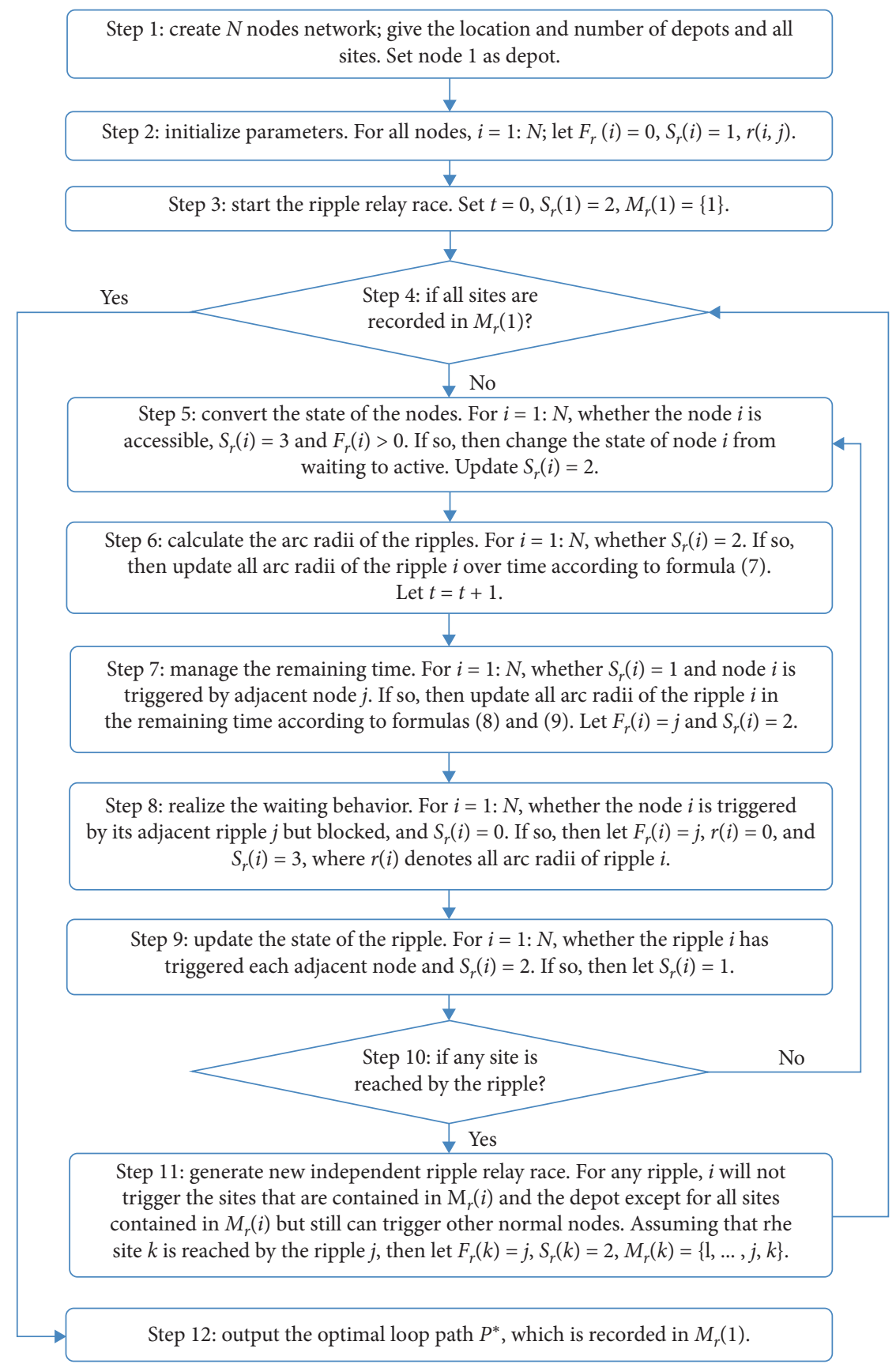

FIgURE 4: The flowchart of the FRSA based on the CEPO methodology.

\section{Experimental Results and Analysis}

4.1. Static Delivery Sequence Determination Tests Compared with GA. In this experiment, to achieve a fair understanding of the performance of FRSA, four tests with different site numbers are conducted to resolve the traditional VRP. A widely used GA is selected to output the results for the comparative analysis. A series of sites (red points) and depot (green point) are generated as follows: the location of the depot is set as the left side as an example and the locations of the sites are randomly distributed in the right district. All nodes have the associated links and connected to their adjacent nodes, and the weights of the links depend on their length. To show the generality of the proposed FRSA in the delivery application scenario, the depot is given only one, while there are four sites scales with $n=10,12,14$, and 16 , respectively. The diagrams of the node connection for all tests are shown in Figure 5.

Since the ripple relay race of FRSA is the time-unitoriented process, it will be terminated once a ripple firstly finds the shortest path in a certain time unit. After that, the FRSA will arrange all the complete routes in that time unit, according to the path length from small to large to select the optimal path. For FRSA, an appropriate spreading speed can 


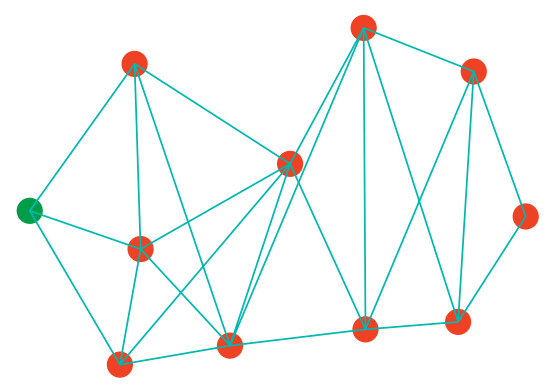

(a)

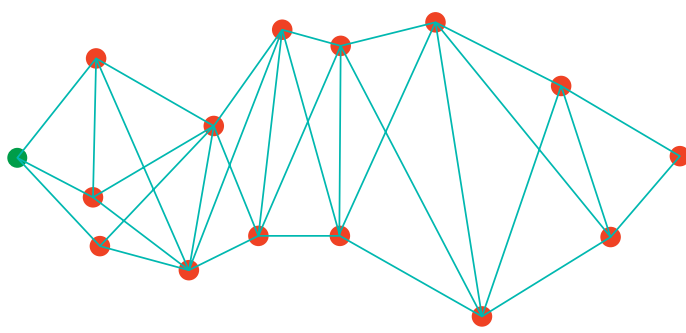

(c)

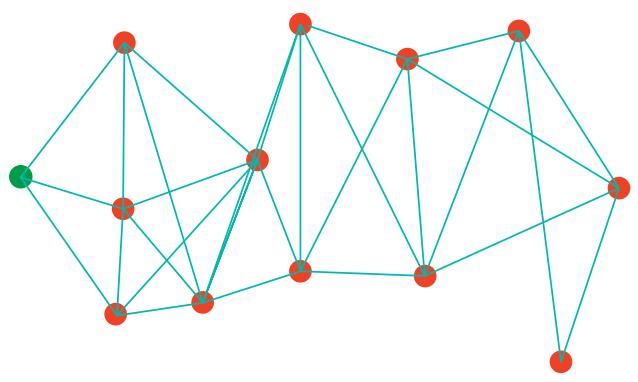

(b)

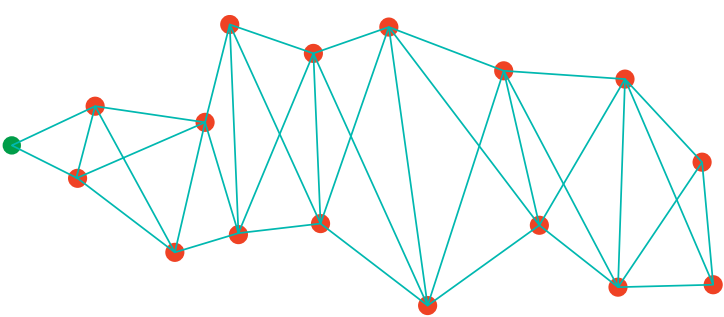

(d)

FIGURE 5: The diagrams of the node connection in different network cases. (a) $n=10$, (b) $n=12$, (c) $n=14$, and (d) $n=16$.

improve the computational efficiency and reduce computation or storage pressures. To ensure the computation efficiency of FRSA optimal, the spreading speed of ripples cannot be too large or too small in the network. If the spreading speed is relatively high, the path length travelled by ripples will be pretty long in a time unit. When the optimal route is solved in a time unit, a great number of travelling trajectories that are closed to the optimal path are likely to be found in the remaining time and still need to be arranged. The unexpected results pose extra work on the calculation module, which means that the computational time required for sorting path length of each complete route increases accordingly. If the spreading speed is relatively low, resulting in more time units required to extend the arc radius of the ripples. The workload of updating the ripple state significantly increases with the growth of time units used in a single run. Hence, the ripple spreading speed can neither be too large nor too small. After comparing multiple spreading speeds through simulated tests, this paper proves that selecting $60 \mathrm{~km} / \mathrm{h}$ has better computing efficiency. Additionally, this speed also conforms to the speed limit management regulations of urban roads in China. As a result, this experiment sets ripple spreading speed $v(i, j)=60 \mathrm{~km} / \mathrm{h}$.

The related results of FRSA and GA are given in Table 1 and Figure 6. One can see that, in terms of PL, it is apparent to observe that FRSA still outperforms GA in all kinds of nodes sizes. The distance of the optimized paths given by FRSA is approximately $33.5 \%$ to $57.7 \%$ smaller than that of GA. Compared with GA, the reduction rate of FRSA reaches the minimal value at $n=12$ and peaks at $n=14$ in terms of PL. One possible reason is that the core technology of FRSA is to globally explore all paths, and eventually ended when the ripple has visited all sites without repetitive activation and returns to the origin. Compared with GA, it
TABLE 1: The optimization results of FRSA and GA in different nodes size scenarios.

\begin{tabular}{lccccc}
\hline & & $n=10$ & $n=12$ & $n=14$ & $n=16$ \\
\hline \multirow{2}{*}{ PL $(\mathrm{km})$} & FRSA & $\mathbf{3 3 . 1 7}$ & $\mathbf{5 1 . 9}$ & $\mathbf{4 5 . 9 6}$ & $\mathbf{5 3 . 9 7}$ \\
& GA & 59.96 & 78.05 & 108.66 & 112.53 \\
\hline \multirow{2}{*}{ CT $(\mathrm{s})$} & FRSA & $\mathbf{2 . 6 3}$ & $\mathbf{7 5 . 0 7}$ & $\mathbf{1 2 8 . 4 5}$ & $\mathbf{1 0 6 2 . 8 2}$ \\
& GA & 0.1 & 0.11 & 0.11 & 0.16 \\
\hline
\end{tabular}

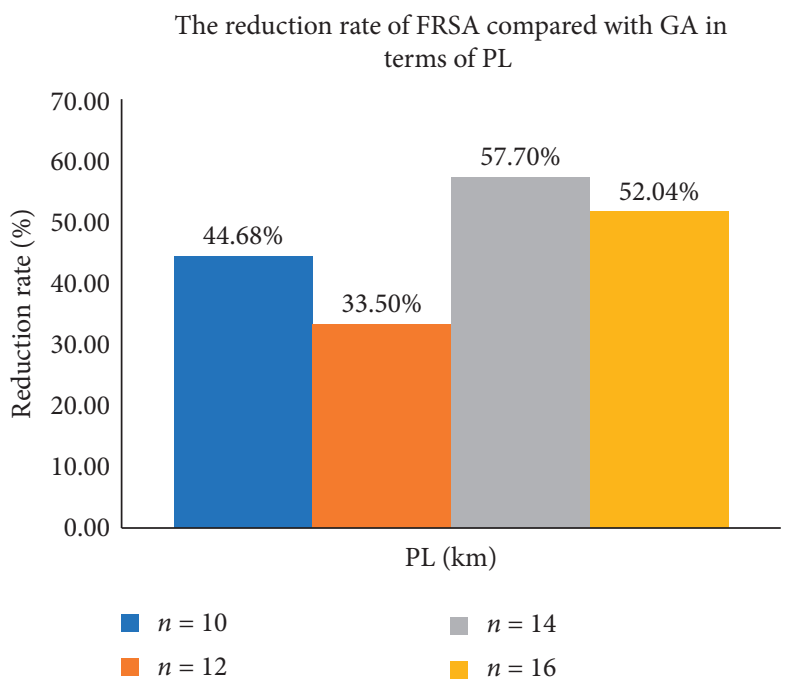

FIGURE 6: The reduction rate of the optimal paths offered by FRSA compared with GA in terms of PL.

possesses better search capacity and will not fall into a local optimum. Unfortunately, as a sacrifice of the huge advantage in solving the best path, the CT of FRSA remarkably goes up with the expansion of node sizes. 
However, unlike other global searching algorithms, FRSA needs no requirements to traversal all results. This is because the optimization steps are divided into a lot of time units, and the ripple relay race will be terminated once the first group of paths is found at a certain time unit. It means that extensive data calculation used for searching other longer loop paths has been omitted. In this respect, FRSA has achieved great progress in terms of CT without losing global searching capacity, although it is relatively large compared with those heuristic algorithms.

\subsection{Delivery Trajectory Optimization Tests considering Dy-} namic Routing Environment. As mentioned in Section 2.1, this study is especially interested in addressing a significant issue via designing an efficient methodology based on CEPO technology, which aims to make the actual delivery trajectory optimal. What makes things difficult is that the simultaneous resolution of the shortest paths between sites and the optimal delivery sequence poses a great challenge on the current methods and tends to be ignored. Therefore, this study investigates the interaction relationship of them and tests the performance of FRSA based on CEPO, comparing to the mainstream heuristic algorithm based on OLRO.

Different from Section 4.1, traffic dynamics of networks are introduced to imitate the actual traffic environment. However, travelling time is significantly affected by traffic flow in urban roads. When the traffic flow of the specific road section is large, the associated vehicle density also is high, which means the space headway between vehicles will be small. However, the smaller space headway between vehicles, along with the more serious disturbance effect between vehicles. This is because the drivers are subject to the front vehicles or the lane-changing behaviour of the adjacent vehicles. The frequency and severity of traffic flow turbulence will be significantly enlarged as the growth of traffic flow, such as dangerous deceleration behaviour, risky lane changes, and sudden accidents. Thus, the negative effects of traffic flow turbulence lead to the reduction of average traffic speed, which poses a direct impact on the increase of travelling time. Conversely, if the traffic flow on the road section is small, the speed of the vehicle tends to be less affected by that, and the travelling time is also reduced accordingly. Thus, travelling time also needs to be an additional assessment indicator, which is used to analyze the temporal efficiency of the optimized routes. In this study, a set of experiments is conducted to verify the promising application of the CEPO-based FRSA in terms of travelling time (TT), path length (PL), and computational time $(\mathrm{CT})$.

To obtain the shortest path between sites in GA, a popular Dijkstra algorithm is introduced to achieve the realization of the OLRO [43-46]. In OLRO, the basic application of the Dijkstra algorithm is to repeat calculating the current shortest path based on the dynamic routing network in each time unit until the vehicle reaches the destination. Based on the current shortest paths between sites, GA is used to find out the optimal delivery sequence via iterative computation and finally presents the complete delivery path. In CEPO, the proposed FRSA will give the optimal delivery trajectory in a single run according to the future routing environment.

In this part, different sizes of urban networks are randomly generated as following: the locations of $N$ nodes are randomly evenly distributed in a rectangular area. There are 4 network scales with $N=64,144,256$, and 400 , respectively. All nodes are connected to their neighbouring nodes, while each node has 3 or 4 connections. The shortest link is less than $150 \mathrm{~m}$. The depot (green point) is always set as the central location of the network, while 8 sites (red points) are distributed around the depot randomly. Furthermore, to achieve the best computational efficiency, $1 \mathrm{~min}$ is selected to represent a time unit. However, the most important thing is to construct the routing environment dynamics, which can be described as traffic speed dynamics:

$$
V_{t+1}(i, j)=V_{t}(i, j)+\sigma_{t}(i, j),
$$

where $\sigma_{t}(i, j)$ denotes the traffic speed variation of the link $(i, j)$ at time $t$.

There are three hypotheses for processing nodes and links, as follows:

(i) To restore the actual traffic operation in the urban network, it is needed to determine the maximum speed and speed variation of the links at each time unit. Suppose $0<V_{t}(i, j) \leq 80 \mathrm{~km} / \mathrm{h}$ and the absolute value of speed variation is less than $4 \mathrm{~km} / \mathrm{h}$ for all links $\left(\left|\sigma_{t}(i, j)\right| \leq 4\right)$ at each time unit.

(ii) Due to the compulsive traffic laws in the signalized intersections, the vehicles must wait at the nodes until they get access to the right to pass through. Thus, the stopping delay caused by waiting at nodes should be contained in travelling time during path optimization. Let $d(i)$ denote the average delay of node $i$, and its value randomly varies from 20 to 60 seconds.

(iii) As a common phenomenon in the urban network, the evolution of the congestion area also should be considered in TDVRP. As shown in Figure 7, the central nodes of congestion areas are evenly distributed in the network and have three kinds of states: spreading, static, and shrinking. It is supposed that the spreading or shrinking congestion areas will expand or narrow in every two minutes, and this action can evolve three times at most. Once the congestion areas spread to the maximum scope, it will keep unchanged in two minutes and then begin to shrink in a regular period.

Some experimental results are clearly given in Table 2 and Figure 8. Table 2 shows the results of the actual delivery trajectories given by FRSA and GA, in terms of TT, PL, and CT. One can see that no matter which scale of the route networks, the proposed FRSA (CEPO-based) outperforms GA (OLRO-based) in terms of TT and PL. From Figure 8, the TT of FRSA is approximately $11.86 \%$ to $20.25 \%$ smaller than that of GA. Moreover, the PL of FRSA is approximately 


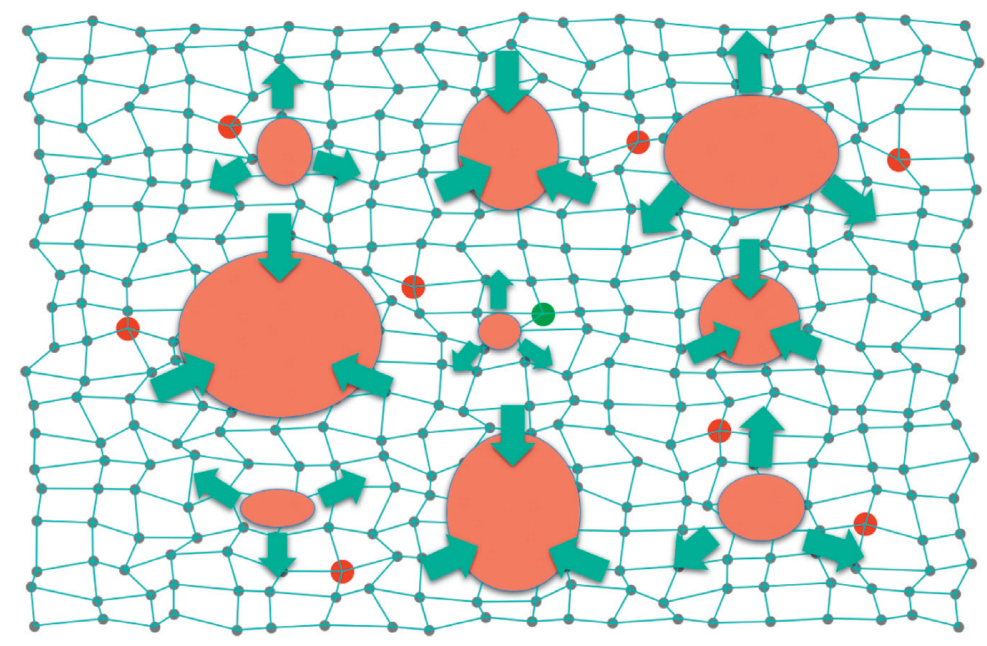

Figure 7: The schematic diagram of evolutionary dynamics for congestion areas $(N=400, n=8)$.

TABLE 2: The optimized results of FRSA and GA in different dynamic networks.

\begin{tabular}{lccccc}
\hline & & $N=64$ & $N=144$ & $N=256$ & $N=400$ \\
\hline \multirow{2}{*}{ TT (min) } & FRSA & $\mathbf{7 0 . 1 8}$ & $\mathbf{9 6 . 9}$ & $\mathbf{1 2 7 . 0 4}$ & $\mathbf{1 5 8 . 9 8}$ \\
& GA & 83.85 & 121.51 & 146.97 & 180.37 \\
\hline \multirow{2}{*}{ PL (km) } & FRSA & $\mathbf{3 9 . 8 2}$ & $\mathbf{4 6 . 8 3}$ & $\mathbf{7 3 . 4 2}$ & $\mathbf{9 2 . 1 1}$ \\
& GA & 40.17 & 64.29 & 88.86 & 97.71 \\
\hline \multirow{2}{*}{ CT (s) } & FRSA & $\mathbf{2 2 5 . 7 4}$ & $\mathbf{3 0 0 . 8 8}$ & $\mathbf{1 4 6 3 . 4 6}$ & $\mathbf{6 0 6 0 . 0 5}$ \\
& GA & 441.45 & 1173.94 & 2269.65 & 6861.33 \\
\hline
\end{tabular}

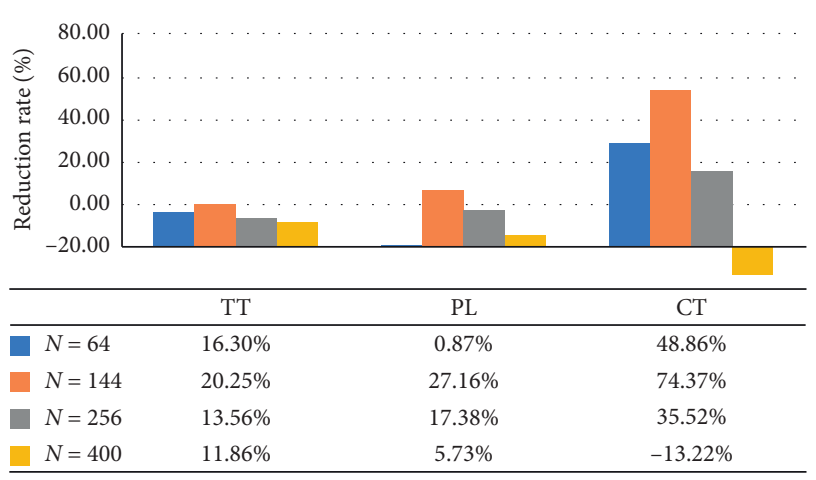

FIGURE 8: The reduction rate of the optimal paths offered by FRSA compared with GA in terms of TT, PL, and CT.

$0.86 \%$ to $27.16 \%$ shorter than that of GA. It is obvious to prove that the large $\mathrm{PL}$ is often inconsistent with the larger TT [34]. In small-size networks $(N=64,144$, and 256), the CT of FRSA is about $35.52 \%$ to $74.37 \%$ smaller than that of GA. Nevertheless, this trend tends to reverses when the network size becomes relatively large, and the CT of FRSA is $13.22 \%$ longer than that of GA when $N=400$.

In terms of TT, PL, and CT, the reduction rate of FRSA gradually boosts from $n=64$, then reaches the peak at $n=144$, and finally declines smoothly over the boost of nodes. Compared with GA, surprisingly, the reduction percentage of FRSA is no-linear growth as the expansion of network size in terms of TT, PL, and CT.

\section{Discussion}

The main goal of this study was to validate the extensive applicability of FRSA based on the framework of CEPO. With this aim, this study performed traditional tests for FRSA and GA in the static networks, as presented in Section 4.1. For the sake of fairness, the reduction rate was used to measure the difference between FRSA and GA in terms of the TT, PL, and CT. The proposed FRSA exhibits superior searching ability and is far better than GA in terms of the cost of the related solutions. However, the encouraging findings also bring corresponding disadvantages. As regards the most serious defect, a massive amount of data is handled in FRSA, along with a sharp increase of CT as the number of sites goes on. An inspiring discovery thing is that, compared to other accurate approaches that achieved a similar effect, FRSA has cut down a tremendous number of computational works without seeking other possible solutions.

As discussed in the previous section, this study aims to propose a commonly used algorithm to address various popular branches of VRP, especially for TDVRP. Therefore, the time-varying traffic environment needs to be considered in testing FRSA as an inevitable development. As a crucial indicator, the TT of the solutions becomes a current focus. In a dynamic routing environment, FRSA is always superior to GA in terms of TT, PL, and CT, except for a special case $(N=400)$ that the CT of FRSA is larger than that of GA. There are three main reasons giving rise to the results that FRSA outperforms the GA in terms of TT and PL. One reason for this is that, in FRSA, the optimal delivery sequence and the shortest path between given OD pairs are solved effectively at the same time. GA just focuses on how to ensure the delivery sequence better, while Dijkstra only concentrates on how to find the best path between OD pairs. The second reason is that FRSA has more advantages to derive the optimal solution via global search, while many heuristic algorithms can only find an approximate solution through parallel computing. The last reason contributes to most to this result, the time-unit-oriented optimization steps of FRSA can coevolve with the changes of routing 
environment and achieve the realization of CEPO. It owns unique features that avoid the coming congestion in advance, or allow waiting behaviour to prevent the wrong detour measure. Therefore, under the coupling effect of three critical factors, the actual travelling trajectory with a shorter TT can be obtained in the nature of things. Except for the big size network like $n=400$, the CT of FRSA is smaller than that of GA in addressing TDVRP. As a result, a smaller CT is spent in FRSA to solve the path optimization in dynamic routing network. But, in $n=400$ network, there are no more advantages for FRSA compared with GA. The main reason contributing to this is that each node provides an opportunity to trigger the ripples, and one node is usually triggered many times during optimization. More nodes also imply the boosting number of ripples will be generated, which asks for a larger CT to update the state transition and ripple spreading situation in a time unit.

However, FRSA possesses some distinct advantages in dealing with VRP, compared with the previous methods. The most outstanding one is that it shows a reliable guarantee of optimality in a time-varying routing environment. The other promising features, like a one-off run before a complicated trip begins and the positive avoidance of congestion areas, also proved that it is superior to the other. These results suggest that, in some urban networks (less than 400 nodes), it would be advisable to execute the FRSA to search the best solution as a practical implementation. As for large network scenarios, it needs to pay attention to whether there is a strict requirement for computational time. If not, FRSA will be a good choice to make the actual travelling trajectory better for addressing the existing VRP.

\section{Conclusions}

This work paves a new way to address various VRP variants, particularly for TDVRP whose main features have been studied for several decades. A mathematical model of FRSA is formulated based on the CEPO technology, which makes use of the future traffic environment to implement path optimization. Due to the effects of traffic flow, network structure, and driver characteristics, some critical factors like timedependent traffic speeds, network topology, temporal congestion area evolution, different vehicle departure times, vehicle waiting behaviour, and network size are considered in the proposed model [47-49]. The objective of the proposed method is to minimize the sum of the delivery costs, such as travelling time and path length. Unlike the existing research studies, this study pays extra attention to explain the internal correlation between determining the delivery sequence and searching the shortest paths between sites, which tends to be ignored. However, FRSA owns several outstanding features, including a congestion avoidance approach, a single run optimization, waiting measure, parallel computing, coevolutionary planning, and global research. The effectiveness of the proposed approach was demonstrated in two sets of simulation experiments. The experimental results show the following: (1) FRSA presents an excellent improvement in reducing actual travelling time and path length, effectively avoiding the wrong detour and regular traffic congestions. It can help to decrease the total distribution cost, fuel consumption, and carbon emissions, which can effectively protect the environment. (2) The optimal delivery sequence and the shortest paths can be derived simultaneously in the dynamic routing networks. This encouraging characteristic is significantly important and beneficial to make the actual travelling trajectory better, which is more likely to be adopted in the practical application compared with other methods. (3) The application of CEPO methodology considering the future routing environment dynamics will decline the travelling time. By contrast, other OLRO-based methods tend to take wrong coping strategies to avoid traffic congestions, which will increase the time and economic costs for logistics companies.

Despite some inspiring results that have been achieved, there still exist some limitations that need to be overcome. Further research may consider the extension of the proposed algorithmic framework to address other VRP variants in special scenarios, by taking into account time window, service time, vehicle capacity, site demand, and pickup. Additionally, the proposed FRSA can be further developed to resolve scalability problems and improve computational efficiency. As a new and effective deterministic algorithm, FRSA opens the door to the development of precise exploration.

\section{Data Availability}

The data used to support the findings of this study are currently under embargo while the research findings are commercialized. Requests for data, 12 months after publication of this article, will be considered by the corresponding author.

\section{Conflicts of Interest}

The authors declare that there are no conflicts of interest regarding the publication of this paper.

\section{References}

[1] X. Jun-xiang and G. Jing-ni, "Research on logistics vehicle routing problem based on big data framework," Journal of Transportation Systems Engineering and Information Technology, vol. 18, no. 1, pp. 86-93, 2018.

[2] M. M. S. Abdulkader, Y. Gajpal, and T. Y. ElMekkawy, "Hybridized ant colony algorithm for the multi compartment vehicle routing problem," Applied Soft Computing, vol. 37, pp. 196-203, 2015.

[3] T. J. Ai and V. Kachitvichyanukul, "A particle swarm optimization for the vehicle routing problem with simultaneous pickup and delivery," Computers \& Operations Research, vol. 36, no. 5, pp. 1693-1702, 2009.

[4] T. J. Ai and V. Kachitvichyanukul, "Particle swarm optimization and two solution representations for solving the capacitated vehicle routing problem," Computers \& Industrial Engineering, vol. 56, no. 1, pp. 380-387, 2009.

[5] S. R. Ait Haddadene, N. Labadie, and C. Prodhon, "A GRASP $\times$ ILS for the vehicle routing problem with time 
windows, synchronization and precedence constraints," Expert Systems with Applications, vol. 66, pp. 274-294, 2016.

[6] S. Akpinar, "Hybrid large neighbourhood search algorithm for capacitated vehicle routing problem," Expert Systems with Applications, vol. 61, pp. 28-38, 2016.

[7] G. B. Dantzig and J. Ramser, "The truck dispatching problem," Management Science, vol. 6, no. 1, pp. 80-91, 1959.

[8] J. J. Alcaraz, L. Caballero-Arnaldos, and J. Vales-Alonso, "Rich vehicle routing problem with last-mile outsourcing decisions," Transportation Research Part E, vol. 129, pp. 263-286, 2019.

[9] C. Lu, G. Kou, X. Zhou, Y. Peng, H. Sheng, and F. E. Alsaadi, "Time-dependent vehicle routing problem with time windows of city logistics with a congestion avoidance approach," Knowledge-Based Systems, vol. 188, pp. 1-8, 2020.

[10] P. Yan, H.-L. Luo, X. Li-ning et al., "A survey of vehicle routing optimization problems and solution methods," Control Theory \& Applications, vol. 36, no. 10, pp. 1573-1584, 2019.

[11] International Energy Agency, $\mathrm{CO}_{2}$ Emissions from Fuel Combustion-Highlights, International Energy Agency, Paris, France, 2015, https://www.iea.org/publications/freepublications/ Publication/ CO2EmissionsFromFuelCombustionHighlights2015pdf.

[12] G. Kou, X. Chao, Y. Peng, F. E. Alsaadi, and E. HerreraViedma, "Machine learning methods for systemic risk analysis in financial sectors," Technological and Economic Development of Economy, vol. 25, no. 5, pp. 716-742, 2019.

[13] C. Duan, C. Deng, A. Gharaei, J. Wu, and B. Wang, "Selective maintenance scheduling under stochastic maintenance quality with multiple maintenance actions," International Journal of Production Research, vol. 56, no. 23, pp. 7160-7178, 2018.

[14] X. Chao, G. Kou, Y. Peng, and F. E. Alsaadi, "Behavior monitoring methods for trade-based money laundering integrating macro and micro prudential regulation: a case from China," Technological and Economic Development of Economy, vol. 25, no. 6, pp. 1081-1096, 2019.

[15] S. A. H. Shekarabi, A. Gharaei, and M. Karimi, "Modelling and optimal lot-sizing of integrated multi-level multiwholesaler supply chains under the shortage and limited warehouse space: generalised outer approximation," International Journal of Systems Science: Operations \& Logistics, vol. 6, no. 3, pp. 237-257, 2018.

[16] S. A. H. Shekarabi, A. Gharaei, and M. Karimi, "Modelling and optimal lot-sizing of the replenishments in constrained, multi-product and bi-objective EPQ models with defective products: generalised cross decomposition," International Journal of Systems Science: Operations \& Logistics, pp. 1-13, 2019.

[17] A. Gharaei, M. Karimi, and S. A. H. Shekarabi, "An integrated multi-product, multi-buyer supply chain under penalty, green, and quality control polices and a vendor managed inventory with consignment stock agreement: the outer approximation with equality relaxation and augmented penalty algorithm," Applied Mathematical Modelling, vol. 69, pp. 223-254, 2019.

[18] A. Gharaei, S. A. Hoseini Shekarabi, and M. Karimi, "Joint economic lot-sizing in multi-product multi-level integrated supply chains: generalized benders decomposition," International Journal of Systems Science: Operations \& Logistics, pp. 1-13, 2019.

[19] N. Rincon-Garcia, B. Waterson, T. J. Cherrett, and F. SalazarArrieta, "A metaheuristic for the time-dependent vehicle routing problem considering driving hours regulations-an application in city logistics," Transportation Research Part A: Policy and Practice, vol. 137, pp. 429-446, 2020.

[20] M. Alinaghian and M. Naderipour, "A novel comprehensive macroscopic model for time-dependent vehicle routing problem with multi-alternative graph to reduce fuel consumption: a case study," Computers \& Industrial Engineering, vol. 99, pp. 210-222, 2016.

[21] C. Malandraki, Time Dependent Vehicle Routing Problem: Formulations, Solution Algorithms and Computations Experiments Northwestern University, Evanston, IL, USA, 1989.

[22] R. Eglese, W. Maden, and A. Slater, "A road timetable to aid vehicle routing and scheduling," Computers \& Operations Research, vol. 33, no. 12, pp. 3508-3519, 2006.

[23] A. V. Donati, R. Montemanni, N. Casagrande, A. E. Rizzoli, and L. M. Gambardella, "Time dependent vehicle routing problem with a multi ant colony system," European Journal of Operational Research, vol. 185, no. 3, pp. 1174-1191, 2008.

[24] A. L. Kok, E. W. Hans, and J. M. J. Schutten, "Vehicle routing under time-dependent travel times: the impact of congestion avoidance," Computers \& Operations Research, vol. 39, no. 5, pp. 910-918, 2012.

[25] Y. Huang, L. Zhao, T. Van Woensel, and J.-P. Gross, “Timedependent vehicle routing problem with path flexibility," Transportation Research Part B: Methodological, vol. 95, pp. 169-195, 2017.

[26] Y. Kuo, "Using simulated annealing to minimize fuel consumption for the time-dependent vehicle routing problem," Computers \& Industrial Engineering, vol. 59, no. 1, pp. 157$165,2010$.

[27] Z.-J. Ma, Y. Wu, and Y. Dai, “A combined order selection and time-dependent vehicle routing problem with time widows for perishable product delivery," Computers \& Industrial Engineering, vol. 114, pp. 101-113, 2017.

[28] D. Taş, N. Dellaert, T. vanWoensel et al., "The time-dependent vehicle routing problem with soft time windows and stochastic travel times," Transportation Research Part C: Emerging Technologies, vol. 48, pp. 66-83, 2014.

[29] M. Andres Figliozzi, "The time dependent vehicle routing problem with time windows: benchmark problems, an efficient solution algorithm, and solution characteristics," Transportation Research Part E: Logistics and Transportation Review, vol. 48, no. 3, pp. 616-636, 2012.

[30] X. Qu, J. Zhang, and S. Wang, "On the stochastic fundamental diagram for freeway traffic: model development, analytical properties, validation, and extensive applications," Transportation Research Part B: Methodological, vol. 104, pp. 256271, 2017.

[31] Y. Bie, X. Xiong, Y. Yan, and X. Qu, "Dynamic headway control for high-frequency bus line based on speed guidance and intersection signal adjustment," Computer-Aided Civil and Infrastructure Engineering, vol. 35, no. 1, pp. 4-25, 2020.

[32] W. Qi, Y. Wang, Y. Bie, and J. Ren, "Prediction model for bus inter-stop travel time considering the impacts of signalized intersections," Transportmetrica A: Transport Science, pp. 119, 2020.

[33] Z. Liu, Y. Liu, Q. Meng, and Q. Cheng, “A tailored machine learning approach for urban transport network flow estimation," Transportation Research Part C: Emerging Technologies, vol. 108, pp. 130-150, 2019.

[34] X.-B. Hu, M.-K. Zhang, Q. Zhang, and J.-Q. Liao, "Co-evolutionary path optimization by ripple-spreading algorithm," Transportation Research Part B: Methodological, vol. 106, pp. 411-432, 2017. 
[35] H. Wen, J. Wu, and Y. Duan, "A methodology of timing coevolutionary path optimization for accident emergency rescue considering future environmental uncertainty," IEEE Access, vol. 7, pp. 131459-131472, 2019.

[36] F. Cheng-hong, Y. Shu-min, and Y. Zhang, "Promoted shortterm traffic flow prediction model based on deep learning and support vector regression," Journal of Transportation Systems Engineering and Information Technology, vol. 19, no. 4, pp. 130-148, 2019.

[37] C. Ru-qing, L. Jia-chun, and Y. Jin-shou, "Short-term traffic flow forecasting based on hybrid FWADE-ELM," Control and Decision, pp. 1-8, 2019.

[38] J. Wang and Q. Shi, "Short-term traffic speed forecasting hybrid model based on chaos-wavelet analysis-support vector machine theory," Transportation Research Part C: Emerging Technologies, vol. 27, pp. 219-232, 2013.

[39] J. Y. T. Wang, M. Ehrgott, and A. Chen, "A bi-objective user equilibrium model of travel time reliability in a road network," Transportation Research Part B: Methodological, vol. 66, pp. 4-15, 2014.

[40] R. Faturechi and E. Miller-Hooks, "Travel time resilience of roadway networks under disaster," Transportation Research Part B: Methodological, vol. 70, pp. 47-64, 2014.

[41] X.-B. Hu and J.-Q. Liao, "Co-evolutionary path optimization by ripple-spreading algorithm," in Proceedings of the 2016 IEEE Congress on Evolutionary Computation, pp. 4535-4542, Canada, Vancouver, July 2016.

[42] X.-B. Hu, M. Wang, M. S. Leeson, E. A. Di Paolo, and H. Liu, "Deterministic agent-based path optimization by mimicking the spreading of ripples," Evolutionary Computation, vol. 24, no. 2, pp. 319-346, 2016.

[43] M. T. Goodrich, Algorithm Design: Foundations, Anal., and Internet Examples, Wiley, Hoboken, NJ, USA, 2001.

[44] M. Sniedovich, "Dijkstra's algorithm revisited: the dynamic programming connexion," Journal of Control and Cybernetics, vol. 35, pp. 599-620, 2006.

[45] M. Sniedovich, Dynamic Programming: Foundations and Principles, Francis \& Taylor, Abingdon, UK, 2010.

[46] S. Russell and P. Norvig, Artificial Intelligence: A Modern Approach, Prentice-Hall, Upper Saddle River, NJ, USA, 3rd edition, 2010.

[47] W. Qi, B. Shen, and L. Wang, "Model of driver's eye movement and ECG index under tunnel environment based on spatiotemporal data," Journal of Advanced Transportation, vol. 2020, Article ID 5215479, 11 pages, 2020.

[48] Z. Liu, S. Wang, K. Huang, J. Chen, and Y. Fu, "Practical taxi sharing schemes at large transport terminals," Transportmetrica B: Transport Dynamics, vol. 7, no. 1, pp. 596-616, 2019.

[49] Y. Liu, Z. Liu, and R. Jia, "DeepPF: a deep learning based architecture for metro passenger flow prediction," Transportation Research Part C: Emerging Technologies, vol. 101, pp. 18-34, 2019. 